\title{
Quantifier Scope and Constituency
}

\author{
Jong C. Park \\ Computer and Information Science \\ University of Pennsylvania \\ 200 South 33rd Street, Philadelphia, PA 19104-6389, USA \\ park@linc.cis.upenn.edu
}

\begin{abstract}
Traditional approaches to quantifier scope typically need stipulation to exclude readings that are unavailable to human understanders. This paper shows that quantifier scope phenomena can be precisely characterized by a semantic representation constrained by surface constituency, if the distinction between referential and quantificational NPs is properly observed. A CCG implementation is described and compared to other approaches.
\end{abstract}

\section{Introduction}

It is generally assumed that sentences with multiple quantified NPs are to be interpreted by one or more unambiguous logical forms in which the scope of traditional logical quantifiers determines the reading or readings. There are two problems with this assumption: (a) without further stipulation there is a tendency to allow too many readings and (b) there is considerable confusion as to how many readings should be allowed arising from contamination of the semantics of many NL quantifiers by referentiality.

There are two well-known techniques for redistributing quantifiers in quantification structures: quantifying-in (Montague, 1974; Cooper, 1983; Keller, 1988; Carpenter, 1994) and quantifier raising (May, 1985). The former provides a compositional way of putting possibly embedded quantifiers to the scope-taking positions, and the latter utilizes a syntactic movement operation at the level of semantics for quantifier placement. There are also approaches that put more emphasis on utilizing contextual information in restricting the generation of semantic forms by choosing a scope-neutral representation augmented with ordering constraints to capture linguistic judgments (Webber, 1979; Kamp, 1981; Heim, 1983; Poesio, 1991; Reyle, 1993). And there are computational approaches that screen unavailable and/or redundant semantic forms (Hobbs \& Shieber, 1987; Moran, 1988; Vestre, 1991). This paper will show that these approaches allow unavaila- ble readings, and thereby miss an important generalization concerning the readings that actually are available.

This paper examines English constructions that allow multiple occurrences of quantified NPs: NP modifications, transitive or ditransitive verbs, that complements, and coordinate structures. Based on a critical analysis of readings that are available from these data, the claim is that scope phenomena can be characterized by a combination of syntactic surface adjacency and semantic function-argument relationship. This characterization will draw upon the old distinction between referential and quantificational NP-semantics (Fodor \& Sag, 1982). We choose to use Combinatory Categorial Grammar to show how surface adjacency affects semantic functionargument relationship, since CCG has the flexibility of composing almost any pair of adjacent constituents with a precise notion of syntactic grammaticality (Steedman, 1990; 1993). ${ }^{1}$

The rest of the paper is organized as follows. First, we discuss in $\$ 2$ how traditional techniques address availability of readings and note some residual problems. Then we give a brief analysis of available readings (\$3), a generalization of the analysis ( $\$ 4)$, and finally describe a computational implementation in Prolog (\$5).

\section{Traditional Approaches}

All three paradigms of grammar formalisms introduced earlier share similar linguistic judgments for their grammaticality analyses. This section examines quantifying-in to show (a) that quantifyingin is a powerful device that allows referential NPinterpretations and (b) that quantifying-in is not sufficiently restricted to account for the available readings for quantificational NP-interpretations.

Quantifying-in is a technique originally introduced to produce appropriate semantic forms for de $r e$ interpretations of NPs inside opaque operators

\footnotetext{
${ }^{1}$ For instance, the result would transfer to Synchronous Tree Adjoining Grammar (Shieber \& Schabes, 1990) without much change.
} 
(Montague, 1974). For example, (a) below has two readings, de re and de dicto, depending on the relativity of the existence of such an individual. They are roughly interpretable as (b) and (c). ${ }^{2}$
(a) John believes that a Republican will win
(b) $\exists r \cdot \operatorname{repub}(r) \wedge$ bel (john, vill $($ vin $(r)))$
(c) bel (john, $\exists r \cdot \operatorname{repub}(r) \wedge$ vill $(\operatorname{vin}(r)))$

(b) has a binder $\exists$ that is quantifying a variable $r$ inside an opaque operator bel, hence the name for the technique. (c) does not have such an intervening operator. Although it is beyond the scope of the present paper to discuss further details of intensionality, it is clear that de re interpretations of NPs are strongly related to referential NP-semantics, in the sense that the de re reading of (a) is about a referred individual and not about an arbitrary such individual. Quantifying-in is designed to make any (possibly embedded) NP take the matrix scope, by leaving a scoped variable in the argument position of the original NP. This would be acceptable for referential NP-semantics.

Montague also proposed to capture purely extensional scope ambiguities using quantifying-in. For example, wide scope reading of a woman in (a) below is accounted for by quantifying-in (with a meaning postulate), patterned after one for (b).
(a) Every man loves a woman.
(b) Every man seeks a white unicorn.

His suggestion is adopted with various subsequent revisions cited earlier. Since any NP, referential or quantificational, requires quantifying-in to outscope another, quantifying-in consequently confounds referential and quantificational NP-semantics. This causes a problem when there is a distributional difference between referential NPs and non-referential NPs, as Fodor \& Sag (1982) have argued, a view which has been followed by the approaches to dynamic interpretation of indefinite NPs cited earlier. It seems hard to reconcile quantifying-in with these observations.

\section{Availability of Readings}

This section proposes a way of sharpening our intuition on available readings and re-examines traditional linguistic judgments on grammatical readings.

While there are undoubted differences in degree of availability among readings dependent upon semantics or discourse preference (Bunt, 1985; Moran, 1988), we will focus on all-or-none structural possibilities afforded by competence grammar. ${ }^{3}$

\footnotetext{
${ }^{2}$ In this simplistic notation, we gloss over tense analysis, among others.

${ }^{3}$ Moran's preference-based algorithm treats certain readings as "highly unpreferred," effectively making them structurally unavailable, from those possible sco-
}

Consider the following unambiguous quantification structure in a generalized quantifier format (hereafter GQ, Barwise \& Cooper, 1981), where quantifier outscopes any quantifiers that may occur in either restriction or body.

(3) quantifier(variable, restriction, body)

Logical forms as notated this way make explicit the functional dependency between the denotations of two ordered quantificational NPs. For example, consider (4) (a) (Partee, 1975). (b) shows one way of representing it in a GQ format.

(4) (a) Three Frenchmen visited five Russians.

(b) three $(f$, frenchuen $(f)$, five $(x$, russians $(r), \operatorname{visited}(f, r))$ )

We can always argue, by enriching the notation, that (4) (b) represents at least four different readings, depending on the particular sense of each involved NP, i.e., group- vs individual-denoting. In every such reading, however, the truth of (4) (b) depends upon finding appropriate individuals (or the group) for $I$ such that each of those individuals (or the group itself) gets associated with appropriate individuals (or a group of individuals) for $r$ via the relation visited. ${ }^{4}$ Notice that there is always a functional dependency of individuals denoted by $x$ upon individuals denoted by $\mathbf{f}$. We claim that this explicit functional dependency can be utilized to test availability of readings. ${ }^{5}$

First, consider the following sentences without coordination.

(5) (a) Two representatives of three companies saw most samples.

(b) Every dealer shows most customers at most three cars.

(c) Most boys think that every man danced with two women.

(a) has three quantifiers, and there are 6 different ways of ordering them. Hobbs \& Shieber (1987) show that among these, the reading in which two representatives outscopes most samples which in turn outscopes three companies is not available from the sentence. They attribute the reason to the logical structure of English as in (3), as it is considered unable to afford an unbound variable, a constraint known as the unbound variable constraint (UVC). ${ }^{6}$ We should note, however, that there is one reading

pings generated by a scheme similar to Hobbs \& Shieber (1887). We claim that competence grammar makes even fewer readings available in the first place.

${ }^{4}$ Without losing generality, therefore, we will consider only individual-denoting NPs in this paper.

${ }^{5}$ Singular NPs such as a company are not helpful to this task since their denotations do not involve multiple individuals which explicitly induce this functional dependency.

${ }^{6}$ The reading would be represented as follows, which has the first occurrence of the variable $c$ left unbound. 
among the remaining five that the UVC allows which in fact does not appear to be available. This is the one in which three companies outscopes most samples which in turn outscopes two representatives (cf. Horn (1972), Fodor (1982)). ${ }^{7}$ This suggests that the UVC may not be the only principle under which Hobbs \& Shieber's reading is excluded. ${ }^{8}$ The other four readings of (a) are self-evidently available. If we generalize over available readings, they are only those that have no quantifiers which intercalate over NP boundaries. ${ }^{9}$

(5) (b) has three quantifiers too, but unlike (5) (a), all the six ways of ordering the quantifiers are available. (5) (c) has only four available readings, where most boys does not intercalate every man and two women. ${ }^{10}$

Consider now sentences including coordination.

(6) (a) Every girl admired, but most boys detested, one of the saxophonists.

(b) Most boys think that every man danced with, but doubt that a few boys talked to, more than two women.

As Geach (1970) pointed out, (a) has only two grammatical readings, though it has three quantifiers. In reading 1 , the same saxophonist was admired and detested at the same time. In reading 2 , every girl admired an arbitrary saxophonist and most boys also detested an arbitrary saxophonist. In particular, missing readings include the one in which every girl admired the same saxophonist and most boys detested the same but another saxophonist. (6) (b)

$t \nabla 0(r, r e p(r) \&$ of $(r, c), \operatorname{most}(s, \operatorname{sanp}(s)$, three $(c, \operatorname{comp}(c), \operatorname{sar}(r, s)))$ )

\footnotetext{
${ }^{7}$ To paraphrase this impossible reading, it is true of a situation under which there were three companies such that there were four samples for each such company such that each such sample was seen by two representatives of that company. Crucially, samples seen by representatives of different companies were not necessarily the same.

${ }^{8}$ This should not be taken as denying the reality of the UVC itself. For example, as one of the referees pointed out, the Uvc is required to explain why, in (a) below, every professor must outscope $a$ friend so as to bind the pronoun his.
}

(a) Most students talked to a friend of every professor about his work.

${ }^{9}$ One can replace most samples with other complex NP such as most samples of at least five products to see this. Certain sentences that apparently escape this generalization will be discussed in the next section.

${ }^{10}$ To see why they are available, it is enough to see that (a) and (b) below have two readings each.

(a) John thinks that every man danced with two women.

(b) Most boys think that Bill danced with two women. also has only two grammatical readings. In one, most boys outscopes every man and a few boys which together outscope more than two women. In the other, more than two women outscopes every man and a few boys, which together outscope most boys.

\section{An Account of Availability}

This section proposes a generalization at the level of semantics for the phenomena described earlier and considers its apparent counterexamples.

Consider a language $\mathcal{L}$ for natural language semantics that explicitly represents function-argument relationships (Jackendoff, 1972). Suppose that in $\mathcal{L}$ the semantic form of a quantified NP is a syntactic argument of the semantic form of a verb or a preposition. (7) through (10) below show well-formed expressions in $\mathcal{C}^{11}$

(7) visited(tive(russian), throe(frenchan))

(8) $\operatorname{san}$ (nost (samp), of (three(conp), two(rep)))

(9) show (three (car), nost (cstmr), every (d1r))

(10) think (^danced (tro(moman), overy(man)), most (boy))

For instance, of has two arguments three (comp) and tro(r॰p), and shor has three arguments.

$\mathcal{L}$ gives rise to a natural generalization of available readings as summarized below. ${ }^{12}$

(11) For a function with $n$ arguments, there are $n$ ! ways of successively providing all the arguments to the function.

This generalization captures the earlier observations about availability of readings. (7), for (4) (a), has two (2!) readings, as visited has two arguments. (8) is an abstraction for four $(2 ! \times 2$ !) readings, as both of and sav have two arguments each. (9) is an abstraction for six (3!) readings, as show has three arguments. Likewise, $(10)$ is an abstraction for four readings.

Coordination gives an interesting constraint on availability of readings. Geach's observation that (6) (a) has two readings suggests that the scope of the object must be determined before it reduces with the coordinate fragment. Suppose that the nonstandard constituent for one of the conjuncts in (6) (a) has a semantic representation shown below.

$$
\lambda x \operatorname{adnired}(x, \operatorname{every}(\operatorname{girl}))
$$

Geach's observation implies that (12) is ambiguous, so that overy (girl) can still take wide (or narrow) scope with respect to the unknown argument. A

\footnotetext{
${ }^{11}$ The up-operator ${ }^{\wedge}$ in (10) takes a term of type $t$ to a term of type e, but a further description of $\mathcal{L}$ is not relevant to the present discussion.

${ }^{12} \mathrm{Nam}$ (1991)'s work is based on a related observation, though he does not make use of the distinction between referential and quantificational NP-semantics.
} 
theory of CCG will be described in the next section to show how to derive scoped logical forms for available readings only.

But first we must consider some apparent counterexamples to the generalization.

(13) (a) Three hunters shot at five tigers.

(b) Every representative of a company saw most samples.

The obvious reading for (a) is called conjunctive or cumulative (Partee, 1975; Webber 1979). In this reading, there are three hunters and five tigers such that shooting events happened between the two parties. Here, arguments are not presented in succession to their function, contrary to the present generalization. Notice, however, that the reading must have two (or more) referential NPs (Higginbotham, 1987). ${ }^{13}$ The question is whether our theory should predict this possibility as well. For a precise notion of availability, we claim that we must appeal to the distinction between referential and quantificational NP-semantics, since almost any referential NP can have the appearance of taking the matrix scope, without affecting the rest of scope phenomena. A related example is (b), where in one reading a referential NP a company arguably outscopes most samples which in turn outscopes every representative (Hobbs \& Shieber, 1987). As we have pointed out earlier, the reading does not generalize to quantified NPs in general.

(14) (a) Some student will investigate two dialects of every language.

(b) Some student will investigate two dialects of, and collect all interesting examples of coordination in, every language.

(c) * Two representative of at least three companies touched, but of few universities saw, most samples.

(a) has a reading in which every language outscopes some student which in turn outscopes two dialects (May, 1985). In a sense, this has intercalating NP quantifiers, an apparent problem to our generalization. However, the grammaticality of (b) opens up the possibility that the two conjuncts can be represented grammatically as functions of arity two, similar to normal transitive verbs. Notice that the generalization is not at work for the fragment of at least three companies touched in (c), since the conjunct is syntactically ungrammatical. At the end of next section, we show how these finer distinctions are made under the CCG framework (See discussion of Figure 5).

${ }^{13}$ For example, (a) below lacks such a reading.

(a) Several men danced with few women.

\section{A CCG Implementation}

This section describes a CCG approach to deriving scoped logical forms so that they range over only grammatical readings.

We will not discuss details of how CCG characterizes natural language syntactically, and refer the interested reader to Steedman (1993). CCGs make use of a limited set of combinators, type raising ( $T$ ), function composition (B), and function substitution (S), with directionality of combination for syntactic grammaticality. For the examples in this paper, we only need type raising and function composition, along with function application. The following shows rules of derivation that we use. Each rule is associated with a label, such as $>$ or $\langle B$ etc, shown at the end.
(a) $X / Y \quad y \quad \Rightarrow x$
(b) $Y \quad X \backslash Y \Rightarrow X$
$(>)$
(c) $X / Y \quad Y / Z \Rightarrow X / Z$
(d) $Y \backslash Z \quad X \backslash Y \Rightarrow X \backslash Z$
(e) np
$\Rightarrow T /(T \backslash$ np $)$
$\Rightarrow T \backslash(T / n p)$

The mapping from syntax to semantics is usually defined in two different ways. One is to use elementary categories, such as np or $\mathbf{s}$, in encoding both syntactic types and logical forms (Jowsey, 1990; Steedman, 1990; Park, 1992). The other is to associate the entire lexical category with a higher-order expression (Kulick, 1995). In this paper, we take the former alternative to describe a first-order rendering of CCG.

Some lexical entries for every are shown below.

(16) $(8: q-\operatorname{every}(X, N, S) /(s: S \backslash n p: X)) / n: X-H$

(17) $(8: \mathrm{s} /(\mathrm{s}: \mathrm{s} \backslash \mathrm{np}: \mathrm{s}$-every $(\mathrm{y}))) / \mathrm{n}: \mathrm{H}$

The information $(s /(s \backslash n p)) / n$ encodes the syntactic fact that every is a constituent which, when a constituent of category $n$ is provided on its right, returns a constituent of category $s /(s \backslash n p)$. q-every $(X, I, S)$ is a term for scoped logical forms. We are using different lexical items, for instance q-overy and s-overy for every, in order to signify their semantic differences. ${ }^{14}$ These lexical entries are just two instances of a general schema for typeraised categories of quantifiers shown below, where $T$ is an arbitrary category.

$$
(T /(T \backslash n p)) / n \text { and }(T \backslash(T / n p)) / n
$$

And the semantic part of (16) and (17) is first-order encoding of (19) (a) and (b), respectively. ${ }^{15}$

\footnotetext{
${ }^{14} \mathrm{q}$-every represents every as a quantifier, and s-every, as a set denoting property. We will use $s$-every $\left(x^{\wedge} \operatorname{man}(x)\right)$ and its $\eta$-reduced equivalent 8 -every (man) interchangeably.

${ }^{15}$ s-quantifier (noun) denotes an arbitrary set $N$ of individuals $d$ such that $d$ has the property noun and that the cardinality of $N$ is determined by quantifier (and
} 

(a) $\lambda n . \lambda P . \forall x \in s$-every $(n) \cdot P(x)$
(b) $\lambda n . \lambda P . P($ s-every $(n))$

(a) encodes wide scope type raising and (b), narrow.

With standard entries for verbs as in (20), logical forms such as (21) and (22) are possible.

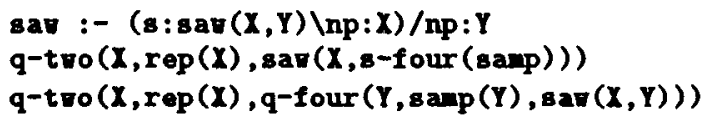

Figure 1 shows different ways of deriving scoped logical forms. In (a), $n: X^{-} \|$unifies with $n: x^{-} g^{-i r l}(x)$, so that gets the value $g^{\operatorname{irl}}(x)$. This value of $x$ is transferred to the expression $s: \operatorname{every}(x, x, s)$ by partial execution (Pereira \& Shieber, 1987; Steedman, 1990; Park, 1992). (a) shows a derivation for a reading in which object NP takes wide scope and (b) shows a derivation for a reading in which subject NP takes wide scope. There are also other derivations.

Figure 2 shows logical forms that can be derived in the present framework from Geach's sentence. Notice that the conjunction forces subject NP to be first composed with the verb, so that subject NP must be type-raised and be combined with the semantics of the transitive verb. As noted earlier, the two categories for the object still make both scope possibilities available, as desired. The following category is used for but.

(23) $((\mathrm{s}:$ and $(P, Q) / \mathrm{np}: X) \backslash(\mathrm{s}: P / \mathrm{np}: X)) /(\mathrm{s}: \mathrm{Q} / \mathrm{np}: \mathrm{X})$

Readings that involve intercalating quantifiers, such as the one where every girl outscopes one saxophonist, which in turn outscopes most boys, are correctly excluded.

Figure 3 shows two different derivations of logical forms for the complex NP two representatives of three companies. (a) shows a derivation for a reading in which the modifying NP takes wide scope and (b) shows the other case. In combination with derivations involving transitive verbs with subject and object NPs, such as ones in Figure 1, this correctly accounts for four grammatical readings for (5) (a). ${ }^{16}$

Figure 4 shows a derivation for a reading, among six, in which most customers outscopes every dealer which in turn outscopes three cars. Some of these readings become unavailable when the sentence contains coordinate structure, such as one below.

Every dealer shows most customers (at most) three cars but most mechanics every car.

noun). We conjecture that this can also be made to capture several related NP-semantics, such as collective NPsemantics and/or referential NP-semantics, though we can not discuss further details here.

${ }^{16}$ As we can see in Figure 3 (a) \& (b), there is no way quantifiers inside $S$ can be placed between the two quantifiers two \& three, correctly excluding the other two readings.
In particular, (24) does not have those two readings in which every dealer intercalates most customers and three cars. This is exactly predicted by the present CCG framework, extending Geach's observation regarding (6) (a), since the coordination forces the two NPs, most customers and three cars, to be composed first (Dowty, 1988; Steedman 1990; Park 1992). (25) through (27) show one such derivation, which results in readings where three cars outscopes most customers but every dealer must take either wide or narrow scope with respect to both most $c u$ stomers and three cars.

(25)

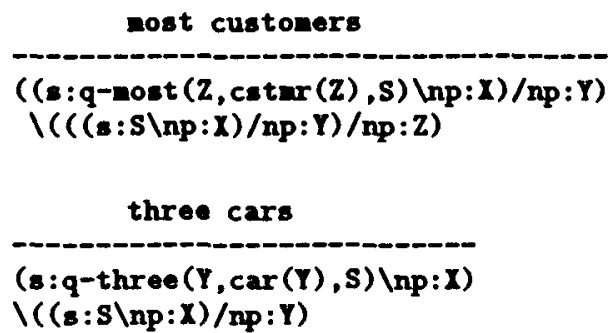

(27)

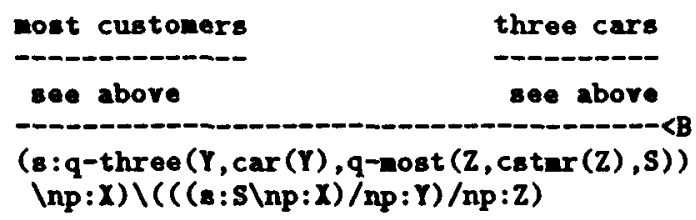

Figure 5 shows the relevant derivation for the fragment investigate two dialects of discussed at end of previous section. It is a conjoinable constituent, but since there is no way of using type-raised category for two for a successful derivation, two dialects can not outscope any other NPs, such as subject NP or the modifying NP (Steedman, 1992). This correctly accounts for our intuition that (14) (a) has an apparently intercalating reading and that (14) (b) has only two readings. However, there is no similar derivation for the fragment of three companies touched, as shown below.

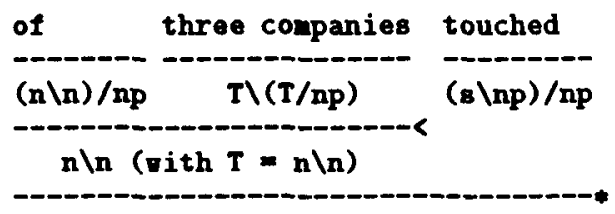

\section{Concluding Remarks}

We have shown that the range of grammatical readings allowed by sentences with multiple quantified NPs can be characterized by abstraction at functionargument structure constrained by syntactic adjacency. This result is in principle available to other paradigms that invoke operations like QR at LF or type-lifting, which are essentially equivalent to abstraction. The advantage of CCG's very free notion 
(a)

\begin{tabular}{|c|c|c|c|}
\hline every & girl & adnired & one saxophonist \\
\hline $\begin{array}{l}\text { s: }: q-\operatorname{every}(X, H, S) \\
/(s: S \backslash n p: X) / n: X-M\end{array}$ & $n: x^{-} \operatorname{girl}(x)$ & $\begin{array}{l}\text { (s: admired }(X, Y) \operatorname{lnp}: X) \\
/ \mathrm{np}: Y\end{array}$ & s:q-one $(Y, \operatorname{sax}(Y), S) \backslash(s: S / n p: Y)$ \\
\hline \multicolumn{4}{|c|}{ s:q-every $(x, g \operatorname{irl}(x), s) /(s: s \backslash$ np: $x)$} \\
\hline s:q-every & , girl $(X)$, adn & $\operatorname{red}(X, Y)) / n p: Y$ & \\
\hline
\end{tabular}

s: $q$-one $(Y, \operatorname{sax}(Y), q$-every $(X, \operatorname{girl}(X, \operatorname{adnired}(X, Y))))$

(b) every girl

a:q-every $(x, g \operatorname{irl}(x), s) /(s: \operatorname{s\backslash np}: x)$ adnired

(a:adired $(X, Y)$ Inp: $X)$

/np:Y one saxophonist

s:S\(s:S/np:s-one (sax))

s : q-overy $(X, g \operatorname{irl}(X)$, admired $(X, Y)) / n p: Y$

$s: q$-every $(X, g i r l(X)$, adnired $(X, 8-o n e(\operatorname{sax})))$

Figure 1: Every girl admired one saxophonist: Two sample derivations

(a) every girl adnired

s:q-every $(X, \operatorname{girl}(X), \operatorname{admired}(X, Y)) / n p: Y$

but most boys detested

$$
-
$$

- - - - - -

one saxophonist

s: S\(s:S/np:s-one(sax))

s: and (q-every $(X, g i r l(X), \operatorname{admired}(X, Y)), q$-most $(X, \operatorname{boy}(X), \operatorname{detested}(X, Y))) / n p: Y$

s: and $(q-\operatorname{erery}(X, g i r l(X), \operatorname{adnired}(x, \operatorname{s-one}(\operatorname{sax}))), q-\operatorname{cost}(x, \operatorname{boy}(X), \operatorname{detested}(x, g-o n e(\operatorname{sax}))))$

(b) every girl adnired but nost boys detested one saxophonist

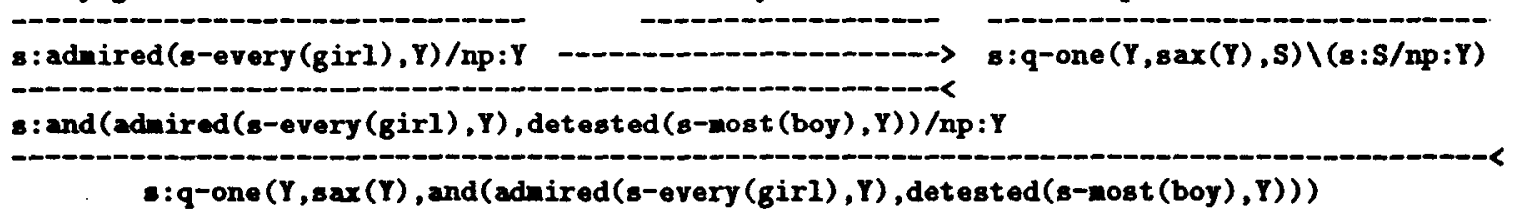

Figure 2: Every girl admired, but most boys detested, one saxophonist: Two sample derivations

$(s: q-t w o(X, X, S)$
$/(s: S \backslash n p: X)) / n: X^{-} \|$

companies

(s:q-three $(C, \operatorname{comp}(C), S 2) /(s: S 1 \backslash n p: x))$

$(s: q-t w o(X$, and $(r e p(X), o f(X, Y)), S) /(s: S \backslash n p: X)) / n p: Y$

$\backslash((s: S 2 /(s: S 1 \operatorname{lnp}: X)) / n p: C)$

$s: q-\operatorname{three}(C, \operatorname{conp}(C), q-t w o(x$, and $(r e p(x)$, of $(x, C)), s)) /(s: s \backslash n p: X)$

(b) two representatives of three companies

$(s: q-t r o(X, X, S) \quad n: X$ and $(\operatorname{rep}(X)$, of $(X, Y)) / n p: Y \quad(s: S 2 /(s: S 1 \backslash n p: X))$
$/(s: S \backslash n p: X)) / n: X-1$

Figure 3: two representatives of three companies: Two sample derivations 


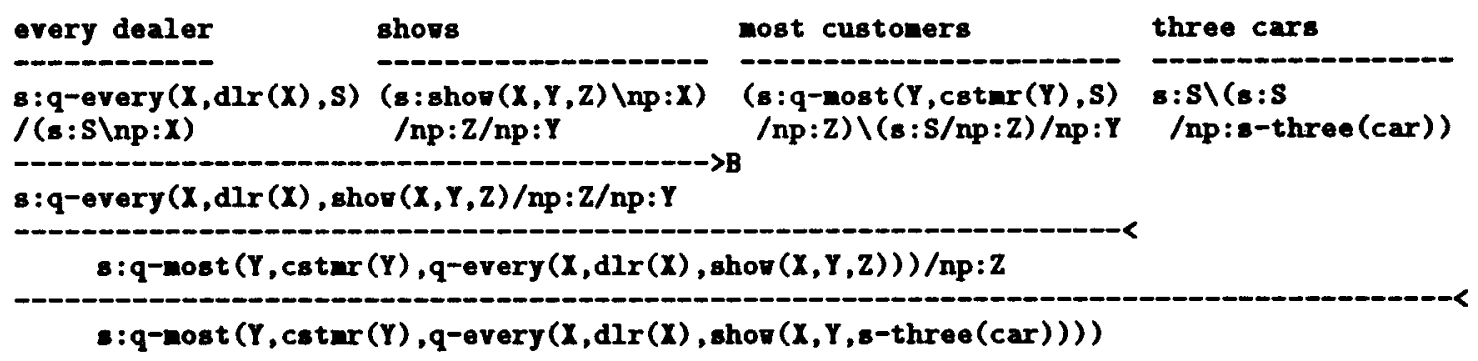

Figure 4: Every dealer shows most customers three cars: One sample derivation

\begin{tabular}{|c|c|c|c|}
\hline investigate & two & dialects & of \\
\hline \multirow[t]{3}{*}{$\begin{array}{l}\text { (s:investigate }(X, Y) \backslash n p: X) \\
\text { /np:Y }\end{array}$} & $\begin{array}{l}\text { np:s-two(I) } \\
/ \mathbf{n}: \mathbf{I}\end{array}$ & $\begin{array}{l}n: H 1 /(n: H 1 \\
\text { ln:Y-dialect }(Y))\end{array}$ & $\begin{array}{l}\left(n: Y^{-} \text {and }(M, \text { of }(Y, Z)) \backslash n: Y^{-} I\right) \\
/ n_{P}: Z\end{array}$ \\
\hline & & $n: Y^{-}$and (dialec & $=t(Y), o f(Y, Z)) / n p: Z$ \\
\hline & $n p: 8-1$ & tro ( $Y^{-}$and (dialect & $(Y), o f(Y, Z))) / n p: Z$ \\
\hline
\end{tabular}

(s: investigate $\left(X, s-t\right.$ tro $\left(Y^{-}\right.$and $(\operatorname{dialect}(Y)$, of $\left.\left.(Y, Z))\right) \backslash n p: X\right) / n p: Z$

Figure 5: investigate two dialects of: One derivation

of surface structure is that it ties abstraction or the equivalent as closely as possible to derivation. Apparent counterexamples to the generalization can be explained by the well-known distinction between referential and quantificational NP-semantics. An implementation of the theory for an English fragment has been written in Prolog, simulating the 2nd order properties.

There is a question of how the non-standard surface structures of CCG are compatible with wellknown conditions on binding and control (including crossover). These conditions are typically stated on standard syntactic dominance relations, but these relations are no longer uniquely derivable once CCG allows non-standard surface structures. We can show, however, that by making use of the obliqueness hierarchy (cf. Jackendoff (1972) and much subsequent work) at the level of LF, rather than surface structure, it is possible to state such conditions (Steedman, 1993).

\section{Acknowledgements}

Special thanks to Mark Steedman. Thanks also to Janet Fodor, Beryl Hoffman, Aravind Joshi, Nobo Komagata, Anthony Kroch, Michael Niv, Charles L. Ortiz, Jinah Park, Scott Prevost, Matthew Stone, Bonnie Webber, and Michael White for their help and criticism at various stages of the presented idea. Thanks are also due to the anonymous referees who made valuable suggestions to clarify the paper. Standard disclaimers apply. The work is supported in part by NSF grant nos. IRI91-17110, and CISE
IIP, CDA 88-22719, DARPA grant no. N660001-94C-6043, and ARO grant no. DAAH04-94-G0426.

\section{References}

Jon Barwise and Robin Cooper. 1981. Generalized quantifiers and natural language. Linguistics $\mathcal{B}$ Philosophy, 5:159 - 219.

Harry C. Bunt. 1985. Mass Terms and ModelTheoretic Semantics. Cambridge University Press.

Bob Carpenter. 1994. A Deductive Account of Scope. The Proceedings of the $13 t h$ West Coast Conference on Formal Linguistics.

Robin Cooper. 1983. Quantification and Syntactic Theory. D. Reidel.

David Dowty. 1988. Type Raising, Functional Composition, and Non-Constituent Conjunction. In Richard T. Oehrle et. al. editors, Categorial Grammars and Natural Language Structures, pages 153 - 197. D. Reidel.

Janet D. Fodor and Ivan A. Sag. 1982. Referential and quantificational indefinites. Linguistics \& Philosophy, 5:355 - 398.

Janet Dean Fodor. 1982. The mental representation of quantifiers. In S. Peters and E. Saarinen, editors, Processes, Beliefs, and Questions, pages 129 - 164. D. Reidel.

Paul T. Geach. 1970. A program for syntax. Synthese, 22:3-17. 
Irene Heim. 1983. File change semantics and the familiarity theory of definiteness. In Rainer Bäuerle et al., editors, Meaning, Use, and the Interpretation of Language. Berlin: de Gruyter.

James Higginbotham. 1987. Indefiniteness and predication. In Eric J. Reuland and Alice G. B. ter Meulen, editors, The Representation of (In)definiteness, pages 43 - 70. MIT Press.

Jerry R. Hobbs and Stuart M. Shieber. 1987. An algorithm for generating quantifier scopings. Computational Linguistics, 13:47 - 63 .

G. M. Horn. 1974. The Noun Phrase Constraint. Ph.D. thesis, University of Massachusetts, Amherst, MA.

Ray S Jackendoff. 1972. Semantic Interpretation in generative grammar. MIT Press.

Einar Jowsey. 1990. Constraining Montague Grammar for Computational Applications. Ph.D. thesis, Department of AI, University of Edinburgh.

Hans Kamp. 1981. A theory of truth and semantic representation. In J. Groenendijk et. al., editor, Formal Methods in the Study of Language. Mathematical Centre, Amsterdam.

William R. Keller. 1988. Nested cooper storage: The proper treatment of quantification in ordinary noun phrases. In E. U. Reyle and E. C. Rohrer, editors, Natural Language Parsing and Linguistic Theories, pages $432-447$. D. Reidel.

Seth Kulick. 1995. Using Higher-Order Logic Programming for Semantic Interpretation of Coordinate Constructs. The Proceedings of the 39rd Annual Meeting of the Association for Computational Linguistics ( $A C L-95$ ).

Robert May. 1985. Logical Form: Its Structure and Derivation. MIT Press.

Richard Montague. 1974. The proper treatment of quantification in ordinary English. In Richmond H. Thomason, editor, Formal Philosophy, pages 247 - 270. Yale University Press.

Douglas B. Moran. 1988. Quantifier scoping in the SRI Core Language Engine. The Proceedings of the 26th Annual Meeting of the Association for Computational Linguistics ( $A C L-88$ ), pages 33 40 .

Seungho Nam. 1991. Scope Interpretation in Nonconstituent Coordination. The Proceedings of the Tenth West Coast Conference on Formal Linguistics, pages $337-348$.

Jong C. Park. 1992. A Unification-Based Semantic Interpretation for Coordinate Constructs. The Proceedings of the 30th Annual Meeting of the Association for Computational Linguistics ( $A C L$ 92), pages $209-215$.
Barbara Partee. 1975. Comments on C. J. Fillmore's and N. Chomsky's papers. In Robert Austerlitz, editor, The Scope of American Linguistics: papers of the first Golden Anniversary Symposium of the Linguistic Society of America. Lisse: Peter de Ridder Press.

Fernando C.N. Pereira and Stuart M. Shieber. 1987. Prolog and Natural-Language Analysis. CSLI Lecture Notes Number 10.

Massimo Poesio. 1991. Scope Ambiguity and Inference. University of Rochester, CS TR-389.

Uwe Reyle. 1993. Dealing with ambiguities by underspecification: Construction, representation and deduction. Journal of Semantics, 10:123 179.

Stuart M. Shieber and Yves Schabes. 1990. Synchronous tree-adjoining grammars. The Proceedings of the 19th International Conference on Computational Linguistics, pages $253-258$.

Mark J. Steedman. 1990. Gapping as constituent coordination. Linguistics $\&$ Philosophy, 13:207 263.

Mark Steedman. 1992. Surface Structure. University of Pennsylvania, Technical Report MS-CIS92-51 (LINC LAB 229).

Mark Steedman. 1993. Categorial grammar: Tutorial overview. Lingua, 90:221 - 258.

Espen J. Vestre. 1991. An algorithm for generating non-redundant quantifier scopings. The Proceedings of the Conference of the European Chapter of the Association for Computational Linguistics, pages $251-256$.

Bonnie Lynn Webber. 1979. A Formal Approach to Discourse Anaphora. Garland Pub. New York. 